\title{
Immediately sequential phacoemulsification performed under topical anaesthesia as day case procedures
}

\author{
M Wertheim, R Burton
}

Br J Ophthalmol 2002;86:1356-1358

Aims: To evaluate the visual outcome, safety, and economic benefits of immediately sequential phacoemulsification performed under topical anaesthesia as a day case procedure.

Methods: Immediately sequential phacoemulsification under topical anaesthesia was performed as a day case procedure on 218 eyes of 109 patients over 21 months by a single senior surgeon using intracameral antibiotics. Case notes were retrospectively analysed.

Results: The final best corrected visual acuity (BCVA) was $6 / 9$ or better in $86 \%$ of eyes with $47 \%$ achieving $6 / 6$ or better. The incidence of intraoperative complications was $0.45 \%$. Postoperative complications occurred in $13.8 \%$ of eyes and included refractive error $>1.00 D$ from refractive aim $(6.9 \%)$. Bilateral complications occurred in $5.5 \%$ of the patients. There were no cases of endophthalmitis. The mean time from surgery to discharge was 24 days.

Conclusion: Immediately sequential phacoemulsification under topical anaesthesia performed as a day case procedure is not associated with an increased number of complications when compared to unilateral phacoemulsification. Under strict surgical protocol with the use of intracameral antibiotics and performed by an experienced surgeon with a good track record this procedure is safe.

B lateral intraocular surgery causes ongoing debate and perpetual disagreement between ophthalmologists. Previously published papers on the subject all comment on its controversial and emotive nature. ${ }^{1-5}$ The main apprehension is the risk of bilateral catastrophic complications such as endophthalmitis.

The currently accepted term of "simultaneous bilateral cataract extraction" is a misnomer for the simple reason that both eyes are not operated on at the same time. Smith and Liu penned the term "immediately sequential cataract surgery" and we have applied this term to our series: "immediately sequential phacoemulsification." Although not performed routinely this type of surgery is becoming more accepted owing to the benefits to the patient, hospital, and society. ${ }^{5}$ In previous reports on this subject strict inclusion criteria have been alluded to. ${ }^{1-5}$ Inclusion criteria for our cohort of patients were not as stringent with a large percentage of patients having immediately sequential phacoemulsification because it was requested by them. We present the first series of patients undergoing immediately sequential phacoemulsification under topical anaesthesia performed as day case procedures.

\section{PATIENTS AND METHODS}

This was a retrospective study of 109 patients (218 eyes) who underwent immediately sequential phacoemulsification (ISP) under topical anaesthesia. The procedures were performed at the Norfolk and Norwich, Cromer, and Norwich BUPA hospitals over a 21 month period from May 1999 to February 2002.
A single senior surgeon performed all the procedures. Data collected included age at operation, indication for bilateral surgery, date of surgery, follow up visits and discharge, preoperative ocular conditions, preoperative refractive error, preoperative and postoperative best corrected visual acuity (BCVA), and intraoperative and postoperative complications.

Patients were offered bilateral surgery at the preoperative visit. Bilateral phacoemulsification was considered in any patient who had a bilateral refractive error (over 2.00 dioptres), or when we felt that one procedure might benefit any patient (for medical, social, or economic reasons) or if any patient requested bilateral surgery. Informed consent was obtained from all patients. Advantages and possible disadvantages of bilateral surgery were fully explained to the patients.

All patients were operated on under topical anaesthesia. The procedures were treated as two separate surgeries in the same session. The operative procedure was rigidly adhered to for each operation. Half an hour preoperatively patients received two drops each of phenylephrine $10 \%$, cyclopentolate $1 \%$, diclofenac $0.1 \%$, and amethocaine $0.1 \%$. In the theatre patients received one drop of povidone-iodine 5\% to the first eye and two drops of amethocaine $0.1 \%$. The patient was then draped, ensuring that the lashes and lid margins were isolated from the eye. The first eye was operated on using a three step corneal incision. The irrigating fluid consisted of a mixture of balanced salt solution (BSS) and gentamicin (2 $\mathrm{mg}$ in $500 \mathrm{ml}$ ) mixed up by the hospital pharmacy. Intracameral lignocaine $0.5 \%$ was instilled in the anterior chamber for 15 seconds before filling the anterior chamber with viscoelastic. After insertion of the intraocular lens $1 \mathrm{mg}$ of vancomycin ( $10 \mathrm{mg}$ per $\mathrm{ml}$ ) was injected into the anterior chamber. The drape was then removed and, while the surgeon rescrubbed, the second eye received a drop of povidineiodine $5 \%$ and two drops of amethocaine $0.1 \%$. The patient was then redraped, infusion fluid change, and a different scrub nurse brought in the new instruments. The same operative procedure was then performed on the second eye. Patients were sent home after surgery and the majority $(65.2 \%)$ were then reviewed the following morning and were given a standard regimen of dexamethasone $0.1 \%$ three times daily to both eyes (one bottle for each eye) for 2 weeks. No topical antibiotic was prescribed. Patients had one more follow up visit that ranged between 2 and 4 weeks. Patients were asked to contact the eye department directly if they noticed decreasing vision, red or painful eyes, or had any concern about either eye.

\section{RESULTS}

A total of 110 consecutive case notes were identified. One case was excluded from the study as the patient was lost to follow up; $72 \%(\mathrm{n}=78)$ were female and $28 \%(\mathrm{n}=31)$ were male; the mean patient age was 79 years (range 47-92 years).

Presumed postoperative anisometropia (if unilateral phacoemulsification was performed) accounted for the majority of the indications for performing bilateral procedures. Fifty five $(49.5 \%)$ patients were operated on because there was a refractive error of over 2.00 dioptres in each eye. In this category, $57 \%$ were myopic with a mean spherical equivalent of -5.93 dioptres (range -2.25 to -15.75 dioptres) and 


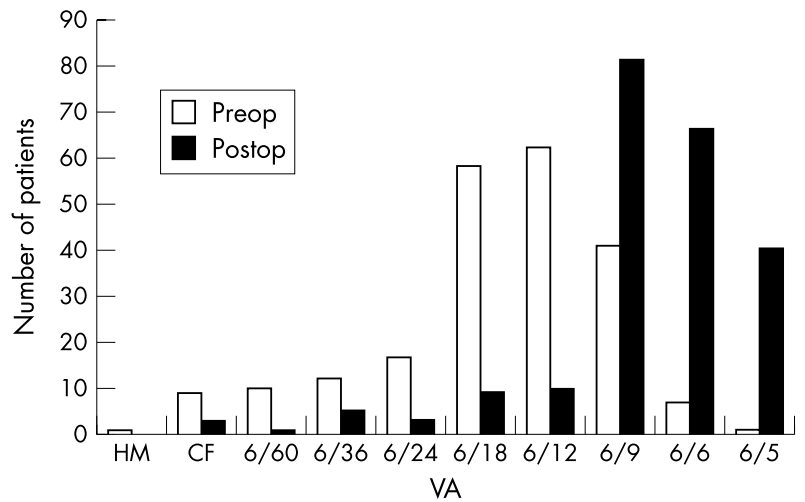

Figure 1 Comparison of preoperative versus postoperative best corrected visual acuity (BCVA).

\begin{tabular}{|ll|}
\hline \multicolumn{2}{|l|}{$\begin{array}{l}\text { Table } 1 \text { Reasons for worse or } \\
\text { unchanged BCVA postoperatively }\end{array}$} \\
\hline $\begin{array}{l}\text { Number } \\
\text { of eyes }\end{array}$ \\
\hline Diagnosis & \\
\hline Coexistent ocular pathology & 2 \\
Age related macular degeneration & 2 \\
Epiretinal membrane & 4 \\
Amblyopia & 1 \\
Retinal detachment & \\
\hline
\end{tabular}

$43 \%$ were hypermetropic with a mean spherical equivalent of +3.99 dioptres (range +2.25 to +8.50 ) .

Preoperative and postoperative BCVA available for 218 eyes is shown in Figure 1. If the 218 eyes are considered individually, $86 \%(n=187)$ achieved a BCVA of $6 / 12$ or better with $47 \%$ $(\mathrm{n}=102)$ achieving $6 / 6$ or better postoperatively; $4.6 \%(\mathrm{n}=$ 10 ) of the eyes had a BCVA that either got worse or did not improve postoperatively (see Table 1).

There was only one intraoperative complication. This complication was a posterior capsule rupture with vitreous loss occurring during irrigation and aspiration in the first operated eye. A three port vitrectomy was immediately performed and an intraocular lens was inserted into the capsular bag. The second eye was then operated on without delay. Both eyes achieved a BCVA of $6 / 9$ at discharge.

The main group of postoperative complications (see Table 2) were "refractive surprises": $6.9 \%(n=15)$ of eyes had this complication. The range of refractive error was between -2.25 to +2.50 in 15 eyes. The single retinal detachment was unfortunately a missed diagnosis preoperatively as a B-scan ultrasound was not performed on this patient with a brunescent cataract. Bilateral complications occurred in 5.5\% $(n=6)$ of patients operated on. There was one case of bilateral cystoid macular oedema-this patient achieved a BCVA of 6/9 in both eyes at discharge. A 92 year old female patient ended up with refractive errors of -5.50 dioptre sphere in each eye postoperatively. Postoperatively this patient had very shallow anterior chambers with almost iridocorneal touch bilaterally. Her intraocular lenses were positioned far too anteriorly thus giving her myopic refractive errors. The cause for this lens position was found to be a rare case of bilateral capsule block syndrome. Bilateral Nd:YAG laser peripheral iridotomies were performed and the patient was treated with atropine $1 \%$.eye drops There was deepening of the anterior chambers and improvement of visual acuities using this conservative treatment alone.

All patients were sent home the day of the bilateral procedure. Initially, patients were reviewed twice-the day after surgery and another follow up appointment 2-3 weeks after surgery. Later in the study the day 1 visit was abandoned and $34.8 \%(n=38)$ of patients had one postoperative visit 2-3 weeks after surgery. The average number of days from surgery to discharge was 24 days (range $1-110$ days).

\section{DISCUSSION}

This is the first reported series of immediately sequential phacoemulsification performed under topical anaesthesia as day case procedures. There have been other reported series ${ }^{1-3}$ of immediately sequential cataract extraction but these studies were of extracapsular cataract extractions (ECCE) performed mainly under general anaesthesia with only a few ECCEs performed under local anaesthesia (mainly peribulbar). Diaper $e t$ al reported a small series of 41 patients ${ }^{4}$ who underwent bilateral phacoemulsification but 40 of these patients were operated on under general anaesthesia.

The major reason for controversy in performing any bilateral intraocular surgery is the risk of bilateral endophthalmitis. We report no cases of unilateral or bilateral endophthalmitis in this study. The UK national endophthalmitis rate is about $0.1 \%{ }^{67}$ and, owing to a paucity of literature on bilateral cataract extraction, the rate for bilateral endophthalmitis cannot be confidently quoted. To reduce the risk of this catastrophic complication we adhered to a strict surgical protocol and administered intracameral antibiotics as described by Gimbel et al in 1994. ${ }^{8}$ Gimbel describes the use of a combination of intracameral antibiotics-namely, gentamicin (active against Gram negative organisms) and vancomycin (active against coagulase negative and positive staphylococci). There were no cases of endophthalmitis in 4684 patients in Gimbel's study. We used gentamicin mixed in the irrigating fluid at a concentration of $2 \mathrm{mg}$ in $500 \mathrm{ml}$ of balanced salt solution and at the end of surgery we injected $1 \mathrm{mg}$ of vancomycin into the anterior chamber. Some groups are concerned about the

\begin{tabular}{|c|c|c|c|c|c|}
\hline & \multirow[b]{2}{*}{ No of eyes } & \multicolumn{4}{|c|}{ Complication rate (\%) } \\
\hline & & This study & Beatty ${ }^{1}$ & Ramsey $^{2}$ & Sharma ${ }^{3}$ \\
\hline Endophthalmitis & 0 & 0 & 0.15 & 0.2 & 0 \\
\hline Cystoid macular oedema & 3 & 1.4 & 1.9 & * & 0.7 \\
\hline Retinal detachment & 0 & 0 & 0 & 0.2 & 0 \\
\hline Refractive (>1.00D) & 15 & 6.9 & * & * & * \\
\hline Uveitis & 1 & 0.5 & 1.4 & 1.9 & 0.7 \\
\hline Raised IOP (>22 mm Hg) & 4 & 1.8 & 3 & 2.9 & 3.8 \\
\hline Corneal oedema & 5 & 2.3 & 0.9 & 1.4 & 3.1 \\
\hline Capsule block syndrome & 2 & 1 & $*$ & $*$ & $*$ \\
\hline \multicolumn{6}{|c|}{$\begin{array}{l}\text { Beatty, } 319 \text { bilateral ECCE. } \\
\text { Ramsey, } 259 \text { bilateral ECCE. } \\
\text { Sharma, } 143 \text { bilateral ECCE and } 1 \text { bilateral phacoemulsification. } \\
{ }^{*} \text { Not mentioned in study. }\end{array}$} \\
\hline
\end{tabular}


Table 3 Postoperative bilateral complications out of 109 patients compared with previous studies

\begin{tabular}{|c|c|c|c|c|c|}
\hline & \multirow{2}{*}{$\begin{array}{l}\text { No of } \\
\text { patients }\end{array}$} & \multicolumn{4}{|c|}{ Complication rate (\%) } \\
\hline & & This study & Beatty ${ }^{1}$ & Ramsey $^{2}$ & Sharma $^{3}$ \\
\hline Refractive & 2 & 1.8 & * & * & * \\
\hline Raised IOP (>22 mm Hg) & 2 & 1.8 & 0.6 & 1.2 & 0 \\
\hline Cystoid macular oedema & 1 & 0.9 & 0 & 0 & 0 \\
\hline Capsule block syndrome & 1 & 0.9 & * & * & * \\
\hline
\end{tabular}

Beatty, 319 bilateral ECCE

Ramsey, 259 bilateral ECCE.

Sharma, 143 bilateral ECCE and 1 bilateral phacoemulsification.

*Not mentioned in study.

potential implications of the wide use of vancomycin. We have found no evidence to support this concern when instilling small amounts of vancomycin into the anterior chamber. The senior surgeon, who performed the ISPs in our study, had no cases of endophthalmitis in 3348 consecutive phacoemulsifications since using Gimbel's method of intracameral antibiotics. We did not use subconjunctival antibiotics in these cases.

Rare complications can occur when performing ISP. These complications should occur as infrequently in bilateral as they do in unilateral cataract extraction. In our study we had two rare complications: one case of bilateral cystoid macular oedema and another of bilateral capsule block syndrome (see Tables 2 and 3). There are no reported incidence rates for bilateral cystoid macular oedema (CMO) and bilateral capsule block syndrome in the literature. The reported incidence of CMO after uncomplicated phacoemulsification is between $0.8 \%$ to $1.2 \%{ }^{9}$ and increases to $12 \%$ in complicated phacoemulsification.

The use of topical anaesthesia for unilateral small incision cataract surgery is well described ${ }^{10-13}$ and does not compromise the safety of surgery. Topical anaesthesia for ISP is the next logical step. In other bilateral cataract studies the reasons for using general anaesthesia over local or topical anaesthesia are not made clear. ${ }^{1-4}$

We sent our patients home immediately after ISP and, to start with, reviewed them the following day. Most other studies have kept immediately sequential cataract extraction patients as inpatients for $1-3$ days, ${ }^{1-4}$ with some patients being followed up at monthly intervals. It has been well described that the day 1 postoperative visit in uncomplicated unilateral cataract extraction could be safely withdrawn. ${ }^{14-16}$ The acute form of endophthalmitis only presents $2-4$ days postoperatively $^{5}$ therefore it would not be picked up on day 1 . The delayed form of endophthalmitis presents usually 5-7 days postoperatively. Somani et al have shown that only $86 \%$ of cases of endophthalmitis present by day 34 postoperatively. ${ }^{17}$ For these reasons we are starting to phase out the day 1 postoperative visit after ISP and are only reviewing patients 2-3 weeks postoperatively. One can warn patients of the ominous signs and symptoms to look out for-namely, decreasing vision, painful eye, redness, discomfort, and so on. We should be able to trust that the patient will seek expert attention if concerned.

Visual function is a complex entity which is a whole of many different parts. Snellen visual acuity makes up only one small dimension of visual function. Laidlaw et al have shown that operating on the second eye gives the patient a significant improvement in visual function. ${ }^{18}$ When one considers patients with refractive errors, the anisometropia after unilateral cataract surgery can be debilitating. Operating on both eyes at once would rid patients of this problem.

With our system of ISP patients might only need visit the hospital three times: a preoperative visit, a visit on the day of surgery, and a postoperative visit $2-3$ weeks later. Because the ISP is being performed under topical anaesthesia there is no longer a need for an anaesthetist.
In conclusion, we do not recommend that all ophthalmology departments adopt this controversial practice. We do however recommend that a surgeon with safe track record and low incidence of infection, intraoperative, and postoperative complications considers ISP under topical anaesthesia performed as a day case procedure. If certain individuals are considered good candidates for ISP we should leave the choice up to the patient who, after a comprehensive discussion of the possible advantages and disadvantages, could make an informed decision.

.................

Authors' affiliations

M Wertheim, R Burton, Department of Ophthalmology, Norfolk and Norwich University Hospital, Colney Lane, Norwich NR4 7UZ, UK

Correspondence to: Michael Wertheim; drwertie@hotmail.com

Accepted for publication 26 June 2002

\section{REFERENCES}

1 Beatty S, Aggarwai RK, David DB, et al. Simultaneous bilateral cataract extraction in the UK. Br J Ophthalmol 1995;79:1111-14.

2 Ramsey AL, Diaper CJM, Saba SN, et al. Simultaneous bilateral cataract extraction. J Cataract Refract Surg 1999;25:753-61.

3 Sharma TK, Worstmann T. Simultaneous bilateral cataract extraction. J Cataract Refract Surg 2001;27:741-4.

4 Diaper CJM, Beirouty ZAY, Saba SN. Simultaneous bilateral phacoemulsification. Eur J Implant Ref Surg 1995;7:232-5.

5 Smith GT, Liu SC. Is it time for a new attitude to "simultaneous" bilateral cataract surgery? Br J Ophthalmol 2001;85: 1489-96.

6 Norregaard JC, Thoning H, Bernth-Petersen P, et al. Risk of endophthalmitis after cataract extraction:results from the International Cataract Surgery Outcomes Audit. Br J Ophthalmol 1997:81:102-06.

7 Desai P, Reidy A, Minassian DC. Profile of patients presenting for cataract surgery in the UK:national data collection. Br J Ophthalmol 1999;83:893-6.

8 Gimbel HV, Sun R, DeBrof BM. Prophylactic intracameral antibiotics during cataract surgery:the incidence of endophthalmitis and corneal endothelial cell loss. Eur J Implant Refract Surg 1994;6:280-5.

9 Royal College of Ophthalmologists. Guidlines for cataract surgery. London: RCO, 1995:10.

10 Claoue C, Lanigan C. Topical anaesthesia for cataract surgery. Aus NZ J Ophthalmol 1997;25:265-8.

11 Duguid IG, Claove CM, Thamby-Rajah Y, et al. Topical anaesthesia for phacoemulsification surgery. Eye 1995;4:456-9

12 Maclean H, Burton T, Murray A. Patient comfort during cataract surgery with modified topical and peribulbar anesthesia. J Cataract Refract Surg 1997:23:277-83

13 Manners TD, Burton RL. Randomised trial of topical versus sub-Tenon's local anesthesia for small-incision cataract surgery. Eye 1996; 10:367-70

14 Cohen VM, Demetria $\mathrm{H}$, Jordan $\mathrm{K}$, et al. First day post-operative review following uncomplicated phacoemulsification. Eye 1998;4:634-6.

15 Whitefield L, Crowston J, Little BC. First day follow up for routine phacoemulsification? Br J Ophthalmol 1996;80:149-50.

16 Tan JHY, Newman DK, Klunker C, et al. Phacoemulsification cataract surgery: Is routine review necessary on the first post-operative day? Eye 2000;14:53-5.

17 Somani S, Grinbaum A, Slomovic SR. Post operative endophthalmitis:incidence, predisposing surgery, clinical course and outcome. Can J Ophthalmol 1997;32:303-10.

18 Laidlaw A, Harrad R. Can second eye cataract be justified? Eye $1993 ; 7: 690-6$. 